\title{
Stability of the Envelope Evolution of a Cold-Fluid Corkscrewing Elliptic Beam in a Uniform-Focusing Magnetic Field
}

\author{
V. Roytershteyn, C. Chen, and R. Pakter
}

\begin{abstract}
The envelope oscillations of a cold-fluid corkscrewing elliptic beam in a uniform-focusing magnetic field are studied. In particular, by linearizing the generalized beam envelope equations, the eigenmodes of small-amplitude envelope oscillations are calculated for a cold-fluid corkscrewing elliptic beam oscillating about its equilibrium. All of the eigenmodes are shown to be stable.
\end{abstract}

Index Terms-Focusing, particle beam stability, space-chargedominated.

\section{INTRODUCTION}

$\mathbf{H}$ IGH-INTENSITY charged-particle beams are widely used in basic scientific research and industries [1], [2]. One important aspect in the research and development of high-intensity beams is the determination of the equilibrium and stability properties of such systems [3], [4]. For the simplest focusing configuration with an applied uniform-focusing magnetic field, it is well known [4] that there exists a wide variety of azimuthally symmetric beam equilibria in which beams propagate axially along the applied magnetic field and rotate azimuthally with certain internal flow velocity profiles. Some of these equilibria are stable in the context of collisionless nonneutral plasmas [4], [5]. Recently, it has been shown [6], [7] that in this focusing configuration, there also exists a new class of cold-fluid corkscrewing elliptic beam equilibria. In the cold-fluid corkscrewing elliptic beam equilibrium, the transverse-beam cross section is elliptic, and it rotates as the beam propagates along the applied magnetic field. The internal flow velocity profile is a combination of both the elliptical-like rotating flow and quadrupole-like flow. Applications of corkscrewing elliptic beams include beam manipulations [8], [9] such as orienting beam ellipses at the interaction point in a high-energy collider [10] or at a heavy ion fusion target [11].

Manuscript received December 16, 2002; revised January 28, 2003. This work was supported in part by the Department of Energy, Office of High Energy and Nuclear Physics under Grant DE-FG02-95ER-40919, and in part by the Department of Energy under a subcontract with Princeton Plasma Physics Laboratory. The work of R. Pakter was supported by Conselho Nacional de Desenvolvimento Científico e Tecnológico, Brazil.

V. Roytershteyn and C. Chen are with the Plasma Science and Fusion Center, Massachusetts Institute of Technology, Cambridge, MA 02139 USA.

R. Pakter is with the Instituto de Fisica, Universidade Federal do Rio Grande do Sul, 91501-970 Porto Alegre, Brazil, and also with the Plasma Science and Fusion Center, Massachusetts Institute of Technology, Cambridge, MA 02139 USA (e-mail: pakter@if.ufrgs.br).

Digital Object Identifier 10.1109/TPS.2003.815819
In this paper, we analyze the envelope oscillations of a coldfluid corkscrewing elliptic beam about its equilibrium in a uniform-focusing magnetic field. In particular, by linearizing the generalized beam envelope equations, we determine the eigenmodes of small-amplitude envelope oscillations of a cold-fluid corkscrewing elliptic beam about its equilibrium, and show that all of the eigenmodes are stable.

The organization of this paper is as follows. In Section II, we review cold-fluid corkscrewing elliptical beam equilibria for an intense charged-particle beam propagating in a uniform-focusing magnetic field and present an alternative representation for the generalized beam envelope equations. In Section III, we show the existence of two branches of equilibrium solutions to the beam envelope equations and study the equilibrium flow characteristics in both branches. In Section IV, we carry out a linear analysis of the perturbations about the equilibrium solutions, determine the eigenmodes of small-amplitude envelope oscillations of a cold-fluid corkscrewing elliptic beam about its equilibrium, and compare with results of the numerical integration of the generalized envelope equations. Conclusions are drawn in Section V.

\section{COLD-FLuid CORKSCREWING ElLIPTICAL BEAM EQUILIBRIA AND GENERALIZED BEAM ENVELOPE EQUATIONS}

In this section, we review cold-fluid corkscrewing elliptical beam equilibria discovered recently [6], [7], concentrating on the case of a uniform-focusing magnetic field. An alternative representation of the generalized beam envelope equations, which proves more suitable for the stability analysis, is presented. Let us consider a thin, continuous, ultrahigh-brightness space-charge-dominated beam propagating with constant axial velocity $\beta_{b} c \hat{\mathbf{e}}_{z}$ through a linear uniform-focusing magnetic field given by

$$
\mathbf{B}_{0}(\mathbf{x})=B_{z 0} \hat{\mathbf{e}}_{z}
$$

where $c$ is the speed of light in vacuum and $B_{z 0}=$ constant.

For an ultrahigh-brightness beam, thermal (emittance) effects are negligibly small compared to space-charge effects, and the beam can be adequately described by cold-fluid equations. In the paraxial approximation, the steady-state cold-fluid equations for time-stationary flow are

$$
\beta_{b} c \frac{\partial}{\partial s} n_{b}+\nabla_{\perp} \cdot\left(n_{b} \mathbf{V}_{\perp}\right)=0
$$




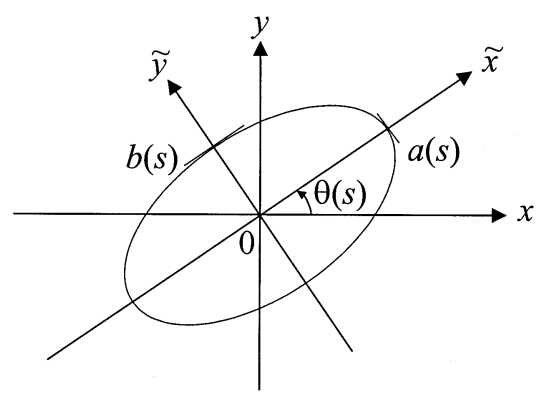

Fig. 1. Equilibrium density profile in the laboratory and rotating coordinate systems.

$$
\begin{aligned}
& \nabla_{\perp}^{2} \phi^{s}=\beta_{b}^{-1} \nabla_{\perp}^{2} A_{z}^{s}=-4 \pi q n_{b} \\
& n_{b}\left(\beta_{b} c \frac{\partial}{\partial s}+\mathbf{V}_{\perp} \cdot \frac{\partial}{\partial \mathbf{x}_{\perp}}\right) \mathbf{V}_{\perp}=\frac{q n_{b}}{\gamma_{b} m} {\left[-\frac{1}{\gamma_{b}^{2}} \nabla_{\perp} \phi^{s}\right.} \\
&\left.+\frac{\mathbf{V}_{\perp}}{c} \times \mathbf{B}_{0}\right]
\end{aligned}
$$

where $m$ and $q$ are the rest mass and charge, respectively, of the beam particles, $s=z=\beta_{b} c t$ is the axial coordinate, $\mathbf{x}_{\perp}=$ $x \hat{\mathbf{e}}_{x}+y \hat{\mathbf{e}}_{y}, \gamma_{b}=\left(1-\beta_{b}^{2}\right)^{-1 / 2}$, and the self-electric and selfmagnetic fields $\mathbf{E}^{s}$ and $\mathbf{B}^{s}$ are determined from the scalar and vector potentials by $\mathbf{E}^{s}=-\nabla_{\perp} \phi^{s}$ and $\mathbf{B}^{s}=\nabla \times A_{z}^{s} \hat{\mathbf{e}}_{z}$. In [6] and [7], it has been shown that there exists a class of solutions to (2)-(4), which describes corkscrewing elliptic beam equilibria for beam propagation through the applied focusing magnetic field defined in (1), in the form

$$
\begin{aligned}
n_{b}\left(\mathbf{x}_{\perp}, s\right)= & \frac{N_{b}}{\pi a(s) b(s)} \Theta\left[1-\frac{\widetilde{x}^{2}}{a^{2}(s)}-\frac{\widetilde{y}^{2}}{b^{2}(s)}\right] \\
\mathbf{V}_{\perp}\left(\mathbf{x}_{\perp}, s\right)= & {\left[\mu_{x}(s) \widetilde{x}-\alpha_{x}(s) \widetilde{y}\right] \beta_{b} c \hat{\mathbf{e}}_{x} } \\
& +\left[\mu_{y}(s) \widetilde{y}+\alpha_{y}(s) \widetilde{x}\right] \beta_{b} c \hat{\mathbf{e}}_{\tilde{y}}^{\imath} .
\end{aligned}
$$

In (5) and (6), $N_{b}$ is the number of particles per unit axial length $\Theta(x)=1$ if $x>0$ and $\Theta(x)=0$ if $x<0$

$$
\begin{aligned}
& \widetilde{x}=x \cos \theta(s)+y \sin \theta(s) \\
& \widetilde{y}=-x \sin \theta(s)+y \cos \theta(s)
\end{aligned}
$$

are the transverse coordinates in a rotating reference frame illustrated in Fig. 1 with $\theta(s)$ as the angle of rotation with respect to the laboratory frame, and the functions $a(s), b(s), \mu_{x}(s), \mu_{y}(s)$, $\alpha_{x}(s), \alpha_{y}(s)$, and $\theta(s)$ are to be determined self consistently.

Substituting (5) and (6) into (2)-(4) leads, after some algebraic manipulations (see [6], [7] for details), to the generalized beam envelope equations

$$
\begin{aligned}
& \frac{d^{2} a}{d s^{2}}-\left(\frac{b^{2}\left(\alpha_{x}^{2}-2 \alpha_{x} \alpha_{y}\right)+a^{2} \alpha_{y}^{2}}{a^{2}-b^{2}}+2 \alpha_{y} \sqrt{\kappa_{z 0}}\right) \times a-\frac{2 K}{a+b}=0 \\
& \frac{d^{2} b}{d s^{2}}+\left(\frac{a^{2}\left(\alpha_{y}^{2}-2 \alpha_{x} \alpha_{y}\right)+b^{2} \alpha_{x}^{2}}{a^{2}-b^{2}}-2 \alpha_{x} \sqrt{\kappa_{z 0}}\right) \times \\
& b-\frac{2 K}{a+b}=0 \\
& \frac{1}{b} \frac{d}{d s}\left\{b^{2}\left[\alpha_{x}+\sqrt{\kappa_{z 0}}\right]\right\}-\frac{a^{3}\left(\alpha_{x}-\alpha_{y}\right)}{a^{2}-b^{2}} \frac{d}{d s}\left(\frac{b}{a}\right)=0
\end{aligned}
$$

$$
\begin{aligned}
\frac{1}{a} \frac{d}{d s}\left\{a^{2}\left[\alpha_{y}+\sqrt{\kappa_{z 0}}\right]\right\}-\frac{b^{3}\left(\alpha_{x}-\alpha_{y}\right)}{a^{2}-b^{2}} \frac{d}{d s}\left(\frac{a}{b}\right) & =0 \\
\frac{d \theta}{d s}-\frac{a^{2} \alpha_{y}-b^{2} \alpha_{x}}{a^{2}-b^{2}} & =0
\end{aligned}
$$

where $\sqrt{\kappa_{z 0}}=q B_{z 0} / 2 \gamma_{b} \beta_{b} m c^{2}$ is the focusing parameter for the uniform-focusing magnetic field and $K=2 q^{2} N_{b} /$ $\gamma_{b}^{3} \beta_{b}^{2} m c^{2}$ is the normalized self-field perveance. The velocity functions $\mu_{x}(s)$ and $\mu_{y}(s)$ are related to $a(s)$ and $b(s)$ by $\mu_{x}=(d a / d s) / a$ and $\mu_{y}=(d b / d s) / b$.

It proves to be convenient to transform (9)-(13) to a more symmetric form by introducing new variables

$$
\begin{aligned}
& x_{1}=a+b \\
& x_{2}=a \alpha_{y}+b \alpha_{x} \\
& x_{3}=\frac{d x_{1}}{d s} \\
& x_{4}=a-b \\
& x_{5}=a \alpha_{y}-b \alpha_{x} \\
& x_{6}=\frac{d x_{4}}{d s} .
\end{aligned}
$$

Adding (9) and (10) and subtracting (10) from (9) yields

$$
\begin{aligned}
\frac{d^{2} x_{1}}{d s^{2}}-2 x_{2} \sqrt{\kappa_{z 0}}-\frac{4 K}{x_{1}}-\frac{x_{2}^{2}}{x_{1}}=0 \\
\frac{d^{2} x_{4}}{d s^{2}}-2 x_{5} \sqrt{\kappa_{z 0}}-\frac{x_{5}^{2}}{x_{4}}=0
\end{aligned}
$$

respectively. Similarly, adding (11) and (12) and subtracting (12) from (11) yields

$$
\begin{aligned}
& \frac{d x_{2}}{d s}+2 \sqrt{\kappa_{z 0}} \frac{d x_{1}}{d s}+\frac{x_{2}}{x_{1}} \frac{d x_{1}}{d s}=0 \\
& \frac{d x_{5}}{d s}+2 \sqrt{\kappa_{z 0}} \frac{d x_{4}}{d s}+\frac{x_{5}}{x_{4}} \frac{d x_{4}}{d s}=0 .
\end{aligned}
$$

Finally, (13) can be expressed in terms of the new variables as

$$
\frac{d \theta}{d s}-\frac{x_{1} x_{5}+x_{2} x_{4}}{2 x_{1} x_{4}}=0 .
$$

Furthermore, it is convenient to express (20)-(23) in terms of the following first-order differential equations:

$$
\begin{aligned}
& \frac{d x_{1}}{d s}=x_{3} \\
& \frac{d x_{2}}{d s}=-2 x_{3} \sqrt{\kappa_{z 0}}-\frac{x_{2} x_{3}}{x_{1}} \\
& \frac{d x_{3}}{d s}=2 x_{2} \sqrt{\kappa_{z 0}}+\frac{4 K}{x_{1}}+\frac{x_{2}^{2}}{x_{1}} \\
& \frac{d x_{4}}{d s}=x_{6} \\
& \frac{d x_{5}}{d s}=-2 x_{6} \sqrt{\kappa_{z 0}}-\frac{x_{5} x_{6}}{x_{4}} \\
& \frac{d x_{6}}{d s}=2 x_{5} \sqrt{\kappa_{z 0}}+\frac{x_{5}^{2}}{x_{4}} .
\end{aligned}
$$

Note that while the generalized envelope (9)-(12) for variables $a(s), b(s), \alpha_{x}(s)$, and $\alpha_{y}(s)$ are all coupled and must be solved simultaneously, the system defined in (25)-(30) decouples into two independent systems with $\left(x_{1}, x_{2}, x_{3}\right)$ and $\left(x_{4}, x_{5}, x_{6}\right)$, 
respectively. In particular, (25)-(27) for $\left(x_{1}, x_{2}, x_{3}\right)$ describe symmetric modes with the envelopes $a$ and $b$ oscillating in phase, whereas (28)-(30) for $\left(x_{4}, x_{5}, x_{6}\right)$ describe antisymmetric modes with the envelopes $a$ and $b$ oscillating with opposite phase. The angle $\theta$ is a slaved variable and can be integrated from (24).

\section{Solution to the BeAm ENVELope EQuations}

The steady-state solutions to (25)-(30) can be obtained analytically. Two branches of physically acceptable special solutions are

$$
x_{1}=-\frac{4 K+x_{2}^{2}}{2 x_{2} \sqrt{\kappa_{z 0}}}=\text { const. } \quad x_{4}=\text { const. } \quad x_{3}=x_{5}=x_{6}=0
$$

for branch A, and

$$
\begin{gathered}
x_{1}=-\frac{4 K+x_{2}^{2}}{2 x_{2} \sqrt{\kappa_{z 0}}}=\text { const. } \quad x_{4}=-\frac{x_{5}}{2 \sqrt{\kappa_{z 0}}}=\text { const. } \\
x_{3}=x_{6}=0
\end{gathered}
$$

for branch B.

It is interesting to point out that these solutions represent two different types of equilibrium flow. Indeed, making use of the definitions in (14)-(19), we can express the equilibrium velocity, (6), as

$$
\frac{\mathbf{V}_{\perp}\left(\mathbf{x}_{\perp}, s\right)}{\beta_{b} c}=\frac{x_{2}}{2}\left(\frac{\widetilde{x}}{a} \hat{\mathbf{e}}_{\tilde{y}}-\frac{\widetilde{y}}{b} \hat{\mathbf{e}}_{x}\right)+\frac{x_{5}}{2}\left(\frac{\widetilde{y}}{b} \hat{\mathbf{e}}_{x}+\frac{\widetilde{x}}{a} \hat{\mathbf{e}}_{\tilde{y}}\right)
$$

In (33), the first term represents elliptical-like rotation, whereas the second term describes quadrupole-like flow. Therefore, $x_{2}$ and $x_{5}$ can be considered as measures of elliptical-like rotation and quadrupole-like flow, respectively. For branch $\mathrm{A}, x_{5}=0$, and the corresponding flow is pure elliptical-like rotation. Branch B is a mixture of both elliptical-like rotation and quadrupole-like flow because both $x_{2}$ and $x_{5}$ are nonzero.

Making use of the definitions in (14)-(19), it is possible to solve the envelope functions $a$ and $b$ in terms of the parameters $\alpha_{x}$ and $\alpha_{y}$ from (31) and (32). This gives

$$
\begin{aligned}
& a=\sqrt{\frac{\alpha_{x}}{\alpha_{y}}}\left[\frac{K}{\kappa_{z 0}-\left(\alpha_{x}+\sqrt{\kappa_{z 0}}\right)\left(\alpha_{y}+\sqrt{\kappa_{z 0}}\right)}\right]^{\frac{1}{2}} \\
& b=\sqrt{\frac{\alpha_{y}}{\alpha_{x}}}\left[\frac{K}{\kappa_{z 0}-\left(\alpha_{x}+\sqrt{\kappa_{z 0}}\right)\left(\alpha_{y}+\sqrt{\kappa_{z 0}}\right)}\right]^{\frac{1}{2}}
\end{aligned}
$$

for branch A, and

$$
\begin{aligned}
& a=\sqrt{\frac{\alpha_{x}+2 \sqrt{\kappa_{z 0}}}{\alpha_{y}+2 \sqrt{\kappa_{z 0}}}}\left[\frac{K}{\kappa_{z 0}-\left(\alpha_{x}+\sqrt{\kappa_{z 0}}\right)\left(\alpha_{y}+\sqrt{\kappa_{z 0}}\right)}\right]^{\frac{1}{2}} \\
& b=\sqrt{\frac{\alpha_{y}+2 \sqrt{\kappa_{z 0}}}{\alpha_{x}+2 \sqrt{\kappa_{z 0}}}}\left[\frac{K}{\kappa_{z 0}-\left(\alpha_{x}+\sqrt{\kappa_{z 0}}\right)\left(\alpha_{y}+\sqrt{\kappa_{z 0}}\right)}\right]^{\frac{1}{2}}
\end{aligned}
$$

for branch B. In both cases, $\alpha_{x}$ and $\alpha_{y}$ are constant. The conditions for the confinement of corkscrewing elliptic beam equilibria are

$$
\frac{\alpha_{x}}{\sqrt{\kappa_{z 0}}}>-2, \frac{\alpha_{y}}{\sqrt{\kappa_{z 0}}}>-2 \text { and }\left(1+\frac{\alpha_{x}}{\sqrt{\kappa_{z 0}}}\right)\left(1+\frac{\alpha_{y}}{\sqrt{\kappa_{z 0}}}\right)<1
$$

In the special case with $\alpha_{x}=\alpha_{y}$, the beam becomes round, recovering the well-known rigid-rotor equilibrium [4], [5].

\section{Stability ANALYsis}

To determine the stability properties of the steady-state solutions in (31) and (32) or (34)-(37), we linearize (25)-(30) to obtain

$$
\frac{d \delta \mathbf{x}}{d s}=\mathbf{A} \cdot \delta \mathbf{x}
$$

where $\boldsymbol{\delta} \mathbf{x}=\left(\delta x_{1}, \delta x_{2}, \ldots, \delta x_{6}\right)^{\mathrm{T}}$ and $\mathbf{A}$ is a $6 \times 6$ matrix with the following nonzero elements:

$$
\begin{aligned}
& A_{13}=1, A_{23}=-2 \sqrt{\kappa_{z 0}}-\frac{x_{2}}{x_{1}} \\
& A_{31}=-\frac{4 K}{x_{1}^{2}}-\frac{x_{2}^{2}}{x_{1}^{2}}, A_{32}=2 \sqrt{\kappa_{z 0}}+\frac{2 x_{2}}{x_{1}} \\
& A_{46}=1, A_{56}=-2 \sqrt{\kappa_{z 0}}-\frac{x_{5}}{x_{4}} \\
& A_{64}=-\frac{x_{5}^{2}}{x_{4}^{2}} \\
& A_{65}=2 \sqrt{\kappa_{z 0}}+\frac{2 x_{5}}{x_{4}} .
\end{aligned}
$$

For the steady-state solutions in (31) and (32) or (34)-(37) to be stable, all of the eigenvalues of $\mathbf{A},\left\{\lambda_{i}\right\}$, must satisfy the condition $\operatorname{Re}\left(\lambda_{i}\right)=0$, where $i=1,2, \ldots, 6$.

\section{A. Eigenvalues for Branch A}

For branch $\mathrm{A}$, the eigenvalue equation $\operatorname{det}(\mathbf{A}-\lambda \mathbf{I})=0$ can be expressed as

$$
\begin{aligned}
& \lambda^{2}\left(\lambda^{2}+4 \kappa_{z 0}\right)\left\{\left(\alpha_{x}+\alpha_{y}\right)^{2} \lambda^{2}+4\left(\alpha_{x}^{2}+\alpha_{y}^{2}\right) \kappa_{z 0}\right. \\
& \left.\quad+8 \alpha_{x} \alpha_{y}\left\lfloor\alpha_{x} \alpha_{y}+\kappa_{z 0}+\left(\alpha_{x}+\alpha_{y}\right) \sqrt{\kappa_{z 0}}\right\rfloor\right\}=0 .
\end{aligned}
$$

Therefore, the eigenvalues are

$$
\begin{aligned}
& \lambda_{1,2}=0 \\
& \lambda_{3,4}= \pm 2 \sqrt{\kappa_{z 0}} i \\
& \lambda_{5,6}= \pm 2 \sqrt{\kappa_{z 0}} \frac{\sqrt{F_{A}}}{\hat{\alpha}_{x}+\hat{\alpha}_{y}} i
\end{aligned}
$$

where $F_{A}=\left(\hat{\alpha}_{x}+\hat{\alpha}_{y}+\hat{\alpha}_{x} \hat{\alpha}_{y}\right)^{2}+\hat{\alpha}_{x}^{2} \hat{\alpha}_{y}^{2}, \hat{\alpha}_{x}=\alpha_{x} / \sqrt{\kappa_{z 0}}$ and $\hat{\alpha}_{y}=\alpha_{y} / \sqrt{\kappa_{z 0}}$. It is readily shown that the function $F_{A}$ has only one real root and, therefore, is always greater or equal to zero, i.e., $F_{A} \geq 0$. Consequently, all of the eigenvalues in (42) satisfy the condition $\operatorname{Re}(\lambda)=0$, which means that branch A is always stable.

A closer examination of the eigenvectors show that the eigenmodes associated with the eigenvalues $\lambda_{3}$ and $\lambda_{4}$ correspond to the envelopes $a$ and $b$ oscillating exactly out of phase, which we refer to as the out-of-phase eigenmode oscillations. On the 

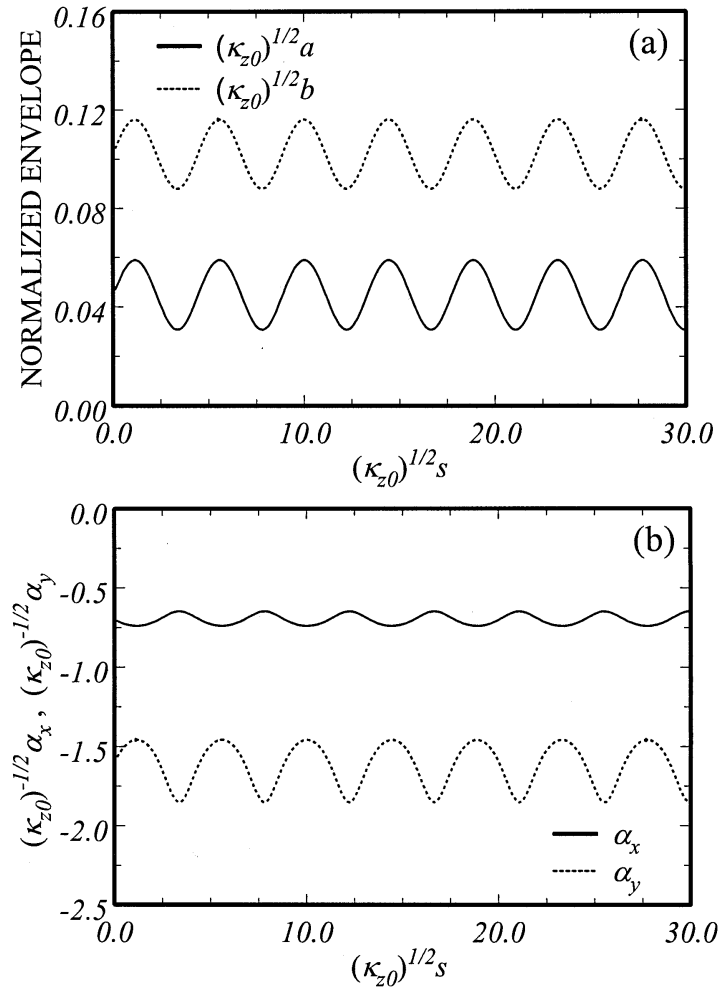

Fig. 2. Plots of the in-phase eigenmode oscillations about an equilibrium solution in branch A. (a) Normalized beam envelopes $a \sqrt{\kappa_{z 0}}$ and $b \sqrt{\kappa_{z 0}}$ versus the normalized propagating distance $s \sqrt{\kappa_{z 0}}$. (b) Normalized variables $\alpha_{x} / \sqrt{\kappa_{z 0}}$ and $\alpha_{x} / \sqrt{\kappa_{z 0}}$ versus the normalized propagating distance $s \sqrt{\kappa_{z 0}}$. Here, the choice of system parameters and initial conditions corresponds to: $K=5.0 \times 10^{-3} ; \alpha_{x}(0) / \sqrt{\kappa_{z 0}}=-0.7 ; \alpha_{y}(0) / \sqrt{\kappa_{z 0}}=-1.6$; $a(0) \sqrt{\kappa_{z 0}}=0.0431 ; b(0) \sqrt{\kappa_{z 0}}=0.0984$; and $a^{\prime}(0)=b^{\prime}(0)=$ 0.02. The equilibrium solution corresponds to: $\alpha_{x}(s) / \sqrt{\kappa_{z 0}}=$ $-0.7 ; \alpha_{y}(s) / \sqrt{\kappa_{z 0}}=-1.6 ; a(s) \sqrt{\kappa_{z 0}}=0.0431 ; b(s) \sqrt{\kappa_{z 0}}=0.0984 ;$ and $a^{\prime}(s)=b^{\prime}(s)=0$

other hand, the eigenmodes associated with the eigenvalues $\lambda_{5}$ and $\lambda_{6}$ correspond to the envelopes $a$ and $b$ oscillating exactly in phase, which we refer to as the in-phase eigenmode oscillations. Furthermore, the vanishing eigenvalues $\lambda_{1}$ and $\lambda_{2}$ correspond to system changes along the equilibrium manifold obtained by continuously varying the constants in (31) and (32) which just lead to neighboring equilibrium solutions and are not relevant to the stability analysis.

Fig. 2 shows the in-phase eigenmode oscillations about an equilibrium solution in branch $\mathrm{A}$, as obtained by integrating (9)-(13) numerically. The choice of system parameters and initial conditions in Fig. 2 corresponds to: $K=5.0 \times 10^{-3}$; $\alpha_{x}(0) / \sqrt{\kappa_{z 0}}=-0.7 ; \alpha_{y}(0) / \sqrt{\kappa_{z 0}}=-1.6 ; a(0) / \sqrt{\kappa_{z 0}}=$ $0.0431 ; b(0) / \sqrt{\kappa_{z 0}}=0.0984 ;$ and $a^{\prime}(0)=b^{\prime}(0)=0.02$. Here, the equilibrium solution corresponds to: $\alpha_{x}(s) / \sqrt{\kappa_{z 0}}=-0.7$; $\alpha_{y}(s) / \sqrt{\kappa_{z 0}}=-1.6 ; a(s) / \sqrt{\kappa_{z 0}}=0.0431 ; b(s) / \sqrt{\kappa_{z 0}}=$ 0.0984 and $a^{\prime}(s)=b^{\prime}(s)=0$. In this case, the envelopes $a$ and $b$ oscillate exactly in phase, but the variables $\alpha_{x}$ and $\alpha_{y}$ oscillate out of phase. The normalized frequency of the eigenmode oscillations is $\left|\lambda_{5}\right| / \sqrt{\kappa_{z 0}}=1.42$, which is in agreement with the expression for the eigenvalue $\lambda_{5}$ (or $\lambda_{6}$ ) given in (42).

Fig. 3 shows the out-of-phase eigenmode oscillations about the same equilibrium solution in branch A, as shown in Fig. 2, as obtained by integrating (9)-(13) numerically. The choice of system parameters and initial conditions in Fig. 3 corresponds
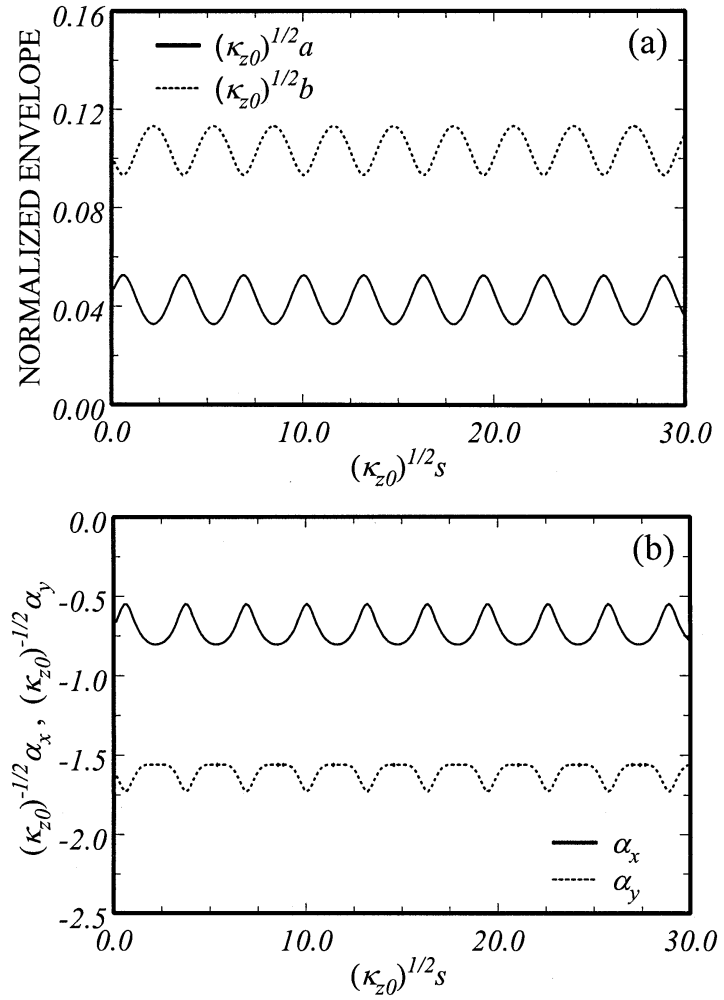

Fig. 3. Plots of the out-of-phase eigenmode oscillations about the same equilibrium solution in branch $\mathrm{A}$ as shown in Fig. 2. (a) Normalized beam envelopes $a \sqrt{\kappa_{z 0}}$ and $b \sqrt{\kappa_{z 0}}$ versus the normalized propagating distance $s \sqrt{\kappa_{z 0}}$. (b) Normalized variables $\alpha_{x} / \sqrt{\kappa_{z 0}}$ and $\alpha_{x} / \sqrt{\kappa_{z 0}}$ versus the normalized propagating distance $s \sqrt{\kappa_{z 0}}$. Here, the choice of system parameters and initial conditions corresponds to: $K=5.0 \times 10^{-3} ; \alpha_{x}(0) / \sqrt{\kappa_{z 0}}=$ $-0.7 ; \alpha_{y}(0) / \sqrt{\kappa_{z 0}}=-1.6 ; a(0) \sqrt{\kappa_{z 0}}=0.0431 ; b(0) \sqrt{\kappa_{z 0}}=0.0984 ;$ and $a^{\prime}(0)=b^{\prime}(0)=0.02$.

to: $K=5.0 \times 10^{-3} ; \alpha_{x}(0) / \sqrt{\kappa_{z 0}}=-0.7 ; \alpha_{y}(0) / \sqrt{\kappa_{z 0}}=$ $-1.6 ; a(0) / \sqrt{\kappa_{z 0}}=0.0431 ; b(0) / \sqrt{\kappa_{z 0}}=0.0984$ and $a^{\prime}(0)=$ $-b^{\prime}(0)=0.02$. For the case shown in Fig. 3, the envelopes $a$ and $b$ oscillate exactly out of phase, and so do the variables $\alpha_{x}$ and $\alpha_{y}$. The normalized frequency of the eigenmode oscillations in Fig. 4 is $\left|\lambda_{3}\right| / \sqrt{\kappa_{z 0}}=2.0$, which is in agreement with the expression for the eigenvalue $\lambda_{3}$ (or $\lambda_{4}$ ) given in (42).

\section{B. Eigenvalues for Branch $B$}

For branch $\mathrm{B}$, the eigenvalue equation is of the form

$$
\begin{gathered}
\lambda^{2}\left(\lambda^{2}+4 \kappa_{z 0}\right) \times \\
\left\{\left(\alpha_{x}+\alpha_{y}+4 \sqrt{\kappa_{z 0}}\right)^{2} \lambda^{2}+5\left(\alpha_{x}^{2}+\alpha_{y}^{2}\right) \kappa_{z 0}+\right. \\
16\left(\alpha_{x}+\alpha_{y}+\sqrt{\kappa_{z 0}}\right) \sqrt{\kappa_{z 0}^{3}}+ \\
2 \alpha_{x} \alpha_{y}\left[\alpha_{x} \alpha_{y}+9 \kappa_{z 0}+3\left(\alpha_{x}+\alpha_{y}\right) \sqrt{\kappa_{z 0}}\right]=0
\end{gathered}
$$

which yields the following eigenvalues

$$
\begin{aligned}
& \lambda_{1,2}=0 \\
& \lambda_{3,4}= \pm 2 \sqrt{\kappa_{z 0}} i \\
& \lambda_{5,6}= \pm 2 \sqrt{\kappa_{z 0}} \frac{\sqrt{F_{B}}}{\left|\hat{\alpha}_{x}+\hat{\alpha}_{y}+4\right|} i
\end{aligned}
$$

where $F_{B}=2 \hat{\alpha}_{x}^{2} \hat{\alpha}_{y}^{2}+6 \hat{\alpha}_{x}^{2} \hat{\alpha}_{y}^{2}+5 \hat{\alpha}_{x}^{2}+6 \hat{\alpha}_{y}^{2} \hat{\alpha}_{x}+18 \hat{\alpha}_{x} \hat{\alpha}_{y}+$ $16 \hat{\alpha}_{x}+5 \hat{\alpha}_{y}^{2}+16 \hat{\alpha}_{y}+16$. The $F_{B}$ function has no real roots, and, therefore, is always greater then zero, i.e., $F_{B}>0$. Consequently, all of the eigenvalues in (44) satisfy the condition 

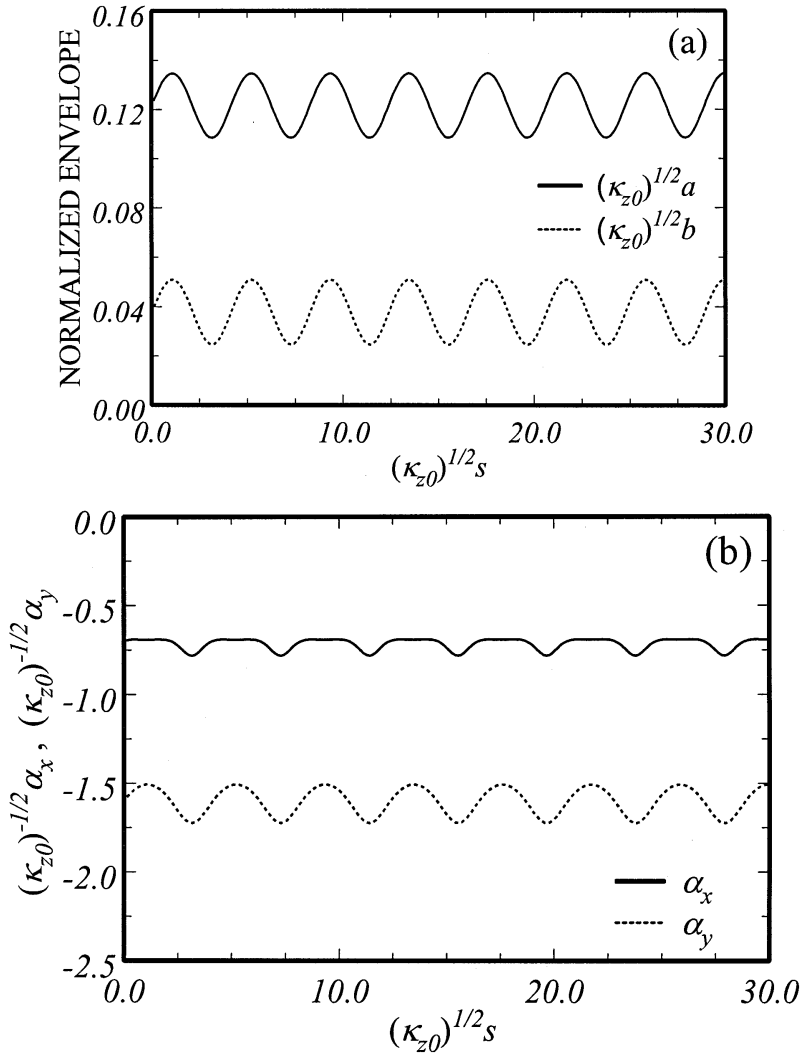

Fig. 4. Plots of the in-phase eigenmode oscillations about an equilibrium solution in branch B. (a) Normalized beam envelopes $a \sqrt{\kappa_{z 0}}$ and $b \sqrt{\kappa_{z 0}}$ versus the normalized propagating distance $s \sqrt{\kappa_{z 0}}$. (b) Normalized variables $\alpha_{x} / \sqrt{\kappa_{z 0}}$ and $\alpha_{x} / \sqrt{\kappa_{z 0}}$ versus the normalized propagating distance $s \sqrt{\kappa_{z 0}}$. Here, the choice of system parameters and initial conditions corresponds to: $K=5.0 \times 10^{-3} ; \alpha_{x}(0) / \sqrt{\kappa_{z 0}}=-0.7 ; \alpha_{y}(0) / \sqrt{\kappa_{z 0}}=-1.6$; $a(0) \sqrt{\kappa_{z 0}}=0.117 ; b(0) \sqrt{\kappa_{z 0}}=0.0361$; and $a^{\prime}(0)=b^{\prime}(0)=$ 0.02 . The equilibrium solution corresponds to: $\alpha_{x}(s) / \sqrt{\kappa_{z 0}}=$ $-0.7 ; \alpha_{y}(s) / \sqrt{\kappa_{z 0}}=-1.6 ; a(s) \sqrt{\kappa_{z 0}}=0.117 ; b(s) \sqrt{\kappa_{z 0}}=0.0361$ and $a^{\prime}(s)=b^{\prime}(s)=0$.

$\operatorname{Re}(\lambda)=0$, which means that branch B is also stable. Associated with the eigenvalues $\lambda_{3}$ and $\lambda_{4}$ in (44) are the in-phase eigenmode oscillations in which the envelopes $a$ and $b$ oscillate exactly in phase, whereas the eigenmodes associated with the eigenvalues $\lambda_{5}$ and $\lambda_{6}$ correspond to the out-of-phase oscillations. The vanishing eigenvalues $\lambda_{1}$ and $\lambda_{2}$ correspond to system changes along the equilibrium manifold defined in (31) and (32), and are not relevant to the stability analysis.

Fig. 4 shows the in-phase eigenmode oscillations about an equilibrium solution in branch $\mathrm{B}$, as obtained by integrating (9)-(13) numerically. The choice of system parameters and initial conditions in Fig. 4 corresponds to: $K=5.0 \times 10^{-3}$; $\alpha_{x}(0) / \sqrt{\kappa_{z 0}}=-0.7 ; \alpha_{y}(0) / \sqrt{\kappa_{z 0}}=-1.6 ; a(0) / \sqrt{\kappa_{z 0}}=$ $0.117 ; ; b(0) / \sqrt{\kappa_{z 0}}=0.0361 ;$ and $a^{\prime}(0)=b^{\prime}(0)=0.02$. Here, the equilibrium solution corresponds to: $\alpha_{x}(s) / \sqrt{\kappa_{z 0}}=-0.7$; $\alpha_{y}(s) / \sqrt{\kappa_{z 0}}=-1.6 ; a(s) / \sqrt{\kappa_{z 0}}=0.117 ; b(s) / \sqrt{\kappa_{z 0}}=$ 0.0361 ; and $a^{\prime}(s)=b^{\prime}(s)=0$. For this eigenmode, the envelopes $a$ and $b$ oscillate exactly in phase, and so do the variables $\alpha_{x}$ and $\alpha_{y}$. The frequency of the oscillations in Fig. 4 is $\left|\lambda_{3}\right| / \sqrt{\kappa_{z 0}}=1.52$, which is in agreement with the expression for the eigenvalue $\lambda_{3}$ (or $\lambda_{4}$ ) given in (44).

Fig. 5 shows the out-of-phase eigenmode oscillations about the same equilibrium solution shown in Fig. 4, as obtained by in-
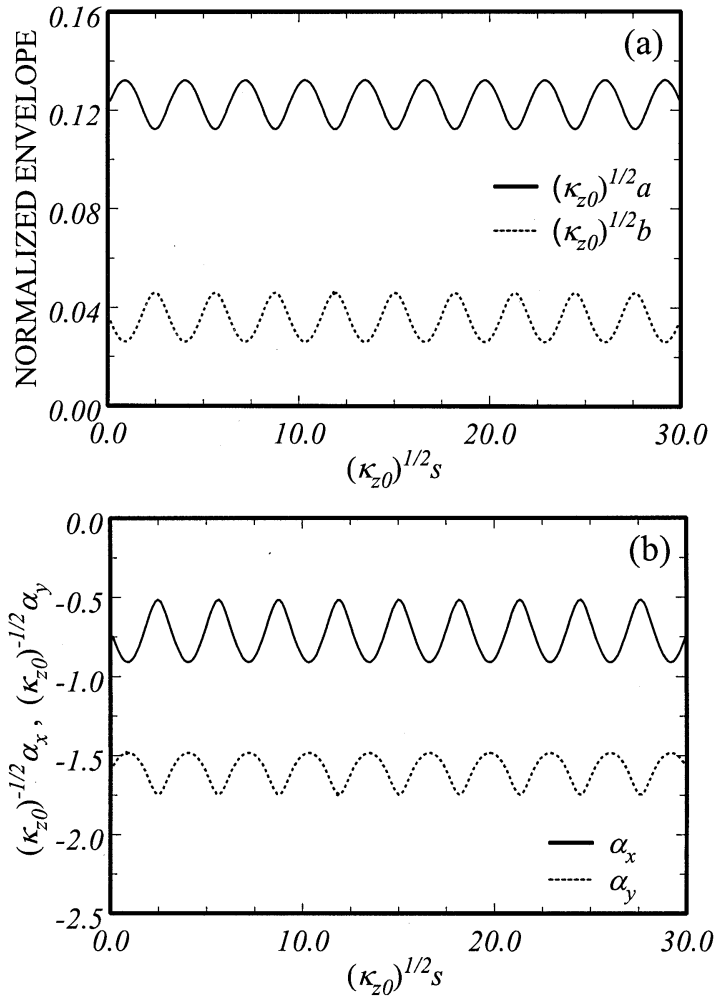

Fig. 5. Plots of the out-of-phase eigenmode oscillations about the same equilibrium solution in branch $\mathrm{B}$ as shown in Fig. 4. (a) Normalized beam envelopes $a \sqrt{\kappa_{z 0}}$ and $b \sqrt{\kappa_{z 0}}$ versus the normalized propagating distance $s \sqrt{\kappa_{z 0}}$. (b) Normalized variables $\alpha_{x} / \sqrt{\kappa_{z 0}}$ and $\alpha_{x} / \sqrt{\kappa_{z 0}}$ versus the normalized propagating distance $s \sqrt{\kappa_{z 0}}$. Here, the choice of system parameters and initial conditions corresponds to: $K=5.0 \times 10^{-3} ; \alpha_{x}(0) / \sqrt{\kappa_{z 0}}=$ $-0.7 ; \alpha_{y}(0) / \sqrt{\kappa_{z 0}}=-1.6 ; a(0) \sqrt{\kappa_{z 0}}=0.117 ; b(0) \sqrt{\kappa_{z 0}}=0.0361 ;$ and $a^{\prime}(0)=-b^{\prime}(0)=0.02$.

tegrating (9)-(13) numerically. The choice of system parameters and initial conditions in Fig. 5 corresponds to: $K=5.0 \times 10^{-3}$; $\alpha_{x}(0) / \sqrt{\kappa_{z 0}}=-0.7 ; \alpha_{y}(0) / \sqrt{\kappa_{z 0}}=-1.6 ; a(0) / \sqrt{\kappa_{z 0}}=$ $0.117 ; b(0) \sqrt{\kappa_{z 0}}=0.0361$; and $a^{\prime}(0)=-b^{\prime}(0)=0.02$. The frequency of the oscillations in Fig. 5 is $\left|\lambda_{5}\right| / \sqrt{\kappa_{z 0}}=2.0$, which in agreement with the expression for the eigenvalue $\lambda_{5}$ (or $\lambda_{6}$ ) given in (44).

To illustrate the previous results, let us consider a concrete example of a high-intensity relativistic electron beam with a current $I_{b}=100 \mathrm{~A}$ and a normalized axial speed $\beta_{b}=0.8$, focused by a uniform magnetic field $B_{z 0}=1.35 \mathrm{kG}$. These parameters correspond to the normalized self-field perveance $K=5.0 \times 10^{-3}$ used in Figs. 2-5 and to a uniform-focusing field parameter $\sqrt{\kappa_{z 0}}=0.297 \mathrm{~cm}^{-1}$. For branch A equilibrium solution discussed in Figs. 2 and 3, the transverse beam density profile is an ellipse with radii $a=1.45 \mathrm{~mm}$ and $b=3.31 \mathrm{~mm}$, and completes a $\theta=2 \pi$ rotation every $s=43.5 \mathrm{~cm}$ of propagation. The periods for stable oscillation about the equilibrium are 14.9 and $10.6 \mathrm{~cm}$ for the in-phase and out-of-phase oscillations, respectively. For branch B equilibrium solution presented in Figs. 4 and 5, $a=3.94$ and $b=1.22 \mathrm{~mm}$, with the beam completing a rotation every $s=12.4 \mathrm{~cm}$ of propagation. In this case, linear theory indicates that the oscillations about the equilibrium occur at length scales comparable to the variation of $\theta$, with periods of $13.9 \mathrm{~cm}$ and $10.6 \mathrm{~cm}$ for the in-phase and out-of-phase oscillations, respectively. 


\section{CONCLUSION}

We have analyzed the envelope oscillations of a cold-fluid corkscrewing elliptic beam in an applied uniform-focusing magnetic field. Two branches of equilibrium solutions to the beam envelope equations were obtained, and the equilibrium flow characteristics in both branches were studied. A linear analysis of the perturbations about the equilibrium solutions was performed to determine the eigenmodes of small-amplitude envelope oscillations of a cold-fluid corkscrewing elliptic beam about its equilibrium. Excellent agreement was found between the eigenmode calculations and the numerical integration of the generalized envelope equations. All of the eigenmodes were shown to be stable.

\section{REFERENCES}

[1] J. Rosenzweig and L. Serafini, Eds., Physics of High-Brightness Beam. New York: World Scientific, 2001.

[2] S. Y. Lee, Ed., Space-Charge Dominated Beams and Application of High-Brightness Beams. New York: AIP, 1996.

[3] I. M. Kapchinskij and V. V. Vladimirskij, "Limitations of proton beam current in a strong focusing linear accelerator associated with the beam space charge," in Proc. Int. Conf. High-Energy Accelerators and Instrumentation. Geneva, Switzerland, 1959, pp. 274-288.

[4] R. C. Davidson, Physics of Nonneutral Plasmas. Reading, MA: Addison-Wesley, 1990.

[5] R. C. Davidson and N. A. Krall, "Vlasov equilibria and stability of an electron gas," Phys. Fluids, vol. 13, pp. 1543-1555, 1970.

[6] R. Pakter and C. Chen, "Cold-fluid equilibrium for a corkscrewing elliptic beam in a variably focusing channel," Phys. Rev. E, Stat. Phys. Plasmas Fluids Relat. Interdiscip. Top., vol. 62, pp. 2789-2796, 2000.
[7] C. Chen and R. Pakter, "Mechanisms and control of beam halo formation in intense microwave sources and accelerators," Phys. Plasmas, vol. 7, pp. 2203-2214, 2000.

[8] D. Chernin, "Evolution of RMS beam envelopes in transport systems with linear X-Y coupling," Part. Accel., vol. 24, pp. 29-44, 1988.

[9] J. J. Barnard, "Emittance growth from rotated quadrupoles in heavy ion accelerators," in Proceedings of the 1995 Particle Accelerator Conference. Piscataway, NJ: IEEE Press, 1995, pp. 3241-3243.

[10] R. H. Siemann, "Overview of linear collider designs," in Proceedings of the 1993 Particle Accelerator Conference. Piscataway, NJ: IEEE Press, 1993, p. 532.

[11] A. W. Molvik and J. J. Barnard, "Matching final focus to distributed radiator target requirements with skew quadrupoles," Nucl. Instrum. Meth. Phys. Res., vol. A464, pp. 271-277, 2001.

V. Roytershteyn is currently working toward the Ph.D. degree at the Plasma Science and Fusion Center, Massachusetts Institute of Technology, Cambridge. His research interest is plasma physics.

C. Chen is a Principal Research Scientist and Leader of the Intense Beam Theoretical Research Group, Plasma Science and Fusion Center, Massachusetts Institute of Technology, Cambridge. His current research interests include plasma physics, beam physics, coherent radiation sources, and nonlinear dynamics and chaos.

R. Pakter is an Associate Professor with the Physics Institute of Universidade Federal do Rio Grande do Sul, Porto Alegre, Brazil. His research interests include beam physics, plasma physics, and nonlinear dynamics and chaos. 